\title{
CONVERGENCE OF FOURIER SERIES EXPANSION RELATED TO FREE GROUPS
}

\author{
G. KUHN ${ }^{1}$
}

\begin{abstract}
In $[0, \pi]$ we consider the complete orthogonal system $P_{n}$ associated to the weight function $\psi=r(2 r-1) \pi^{-1} \sin ^{2} \theta\left(r^{2}-(2 r-1) \cos ^{2} \theta\right)^{-1}$ and we study mean and pointwise convergence of series expansions with respect to the system $P_{n}$ in $L^{p}([0, \pi], d \psi)$. This weight function, and the corresponding system $P_{n}$ arise from the study of Gelfand transforms of radial functions on a finitely generated free group $F_{r}$ and our results can be interpreted in terms of multipliers theory on $F_{r}$.
\end{abstract}

1. Introduction. Harmonic analysis on finitely generated free groups has been recently investigated by several authors $[\mathbf{1}, \mathbf{3}-\mathbf{5}, \mathbf{9 - 1 1}, \mathbf{2 0}]$. In particular, FigáTalamanca and Picardello studied the representation theory for the free group on $r$ generators $F_{r}$ in analogy with the representation theory for $\mathrm{SL}_{2}(R)$. Exploiting the analogy between $K$-bi-invariant functions on $\mathrm{SL}_{2}(R)$ and radial functions on $F_{r}$, they were able to define spherical functions, the Poisson kernel and the principal and complementary series on $F_{r}$ (see also $[\mathbf{2}, \mathbf{1 4}]$ ). Our main reference is [9], to which we refer for all unexplained notions and results.

Denote by $C_{\lambda, R}^{*}$ the closure in the $l^{2}$-convolutor norm of the algebra of all finitely supported radial functions. $C_{\lambda, R}^{*}$ is isomorphic (via the Gelfand transform that we denote by $\widehat{)}$ to the algebra $C(I)$ of all continuous functions on $I=[0, \pi]$. If

$$
d \psi(\theta)=r(2 r-1) \pi^{-1} \sin ^{2} \theta\left(r^{2}-(2 r-1) \cos ^{2} \theta\right)^{-1} d \theta
$$

then the Gelfand transform extends to an isometric isomorphism between $l_{\text {radial }}^{2}\left(F_{r}\right)$ and $L^{2}(I, d \psi)$. There is a complete orthogonal polynomial system $\left\{P_{n}\right\}_{0}^{\infty}$ associated to $d \psi$ (see e.g. [4]):

$$
P_{n}=(2 r-1)^{n / 2}\left\{Y_{n}-(2 r-1)^{-1} Y_{n-2}\right\}
$$

$$
\text { where } Y_{n}=\left\{\begin{array}{l}
\sin ((n+1) \theta)(\sin \theta)^{-1} \\
0 \text { otherwise. }
\end{array}\right.
$$

(We notice that $P_{n}$ is a linear combination of two Gegenbauer polynomials $C_{n}^{\mathbf{1}}[\mathbf{7}]$, or, equivalently, characters on $\mathrm{SU}(2)$.)

In this paper we study the convergence (norm and pointwise) of series expansions with respect to the system $P_{n}$ of functions belonging to $L^{p}(I, d \psi)$. Our results (see the Theorem in $\S 3$ ) resemble those concerning norm and pointwise convergence of series expansions with respect to the Gegenbauer polynomials $C_{n}^{1}$ or, equivalently, Fourier expansions of central functions on $\mathrm{SU}(2)[6,16-18,21]$. This suggests

Received by the editors June 28, 1983.

1980 Mathematics Subject Classification. Primary 43A50.

${ }^{1}$ This research was supported by Ministero P. I. 
an analogy between radial harmonic analysis on $F_{r}$ and central harmonic analysis on $\mathrm{SU}(2)$. Finally, we notice that our results can also be interpreted in terms of multipliers theory on $F_{r}$.

2. Notation. We shall denote by $\chi_{n}$ the characteristic function of the set $E_{n}=\left\{x \in F_{r}:|x|=n\right\}$ and set $\omega=(2 r-1)^{1 / 2}, a=(2 r-1)^{1 / 2}(2 r)^{-1 / 2}, b=$ $(2 r(2 r-1))^{-1 / 2}$. From $[1,13,15]$ we know that the Gelfand transform extends to an isometric isomorphism between $L^{1}(I, d \psi)$ and the subspace of radial elements of the Fourier algebra of $F_{r}$ so that $\hat{f}$ will denote any function in $L^{p}(I, d \psi)(1 \leq p \leq \infty)$ while $f$ will be the corresponding radial function on $F_{r} ;\|\cdot\|_{p}$ will always denote the norm in $L^{p}(I, d \psi)$. According with the hypergroup structure of $I$ [15] we shall define a "convolution" by the formula

$$
\hat{f} * \hat{g}=(f \cdot g)^{\wedge} \text { for any } \hat{f} \in L^{1}(I, d \psi), \hat{g} \in L^{p}(I, d \psi)(1<p<\infty) .
$$

Since $d \psi$ is an invariant measure on $I$ (with respect to the hypergroup structure of I) we get

$$
\begin{gathered}
\|\hat{f} * \hat{g}\|_{1} \leq\|\hat{f}\|_{1} \cdot\|\hat{g}\|_{1} \quad \text { for every } \hat{g}, \hat{f} \in L^{1}(I, d \psi), \\
\|\hat{f} * \hat{g}\|_{\infty} \leq\|\hat{f}\|_{1} \cdot\|\hat{g}\|_{\infty} \quad \text { for every } \hat{f} \in L^{1}(I, d \psi), \hat{g} \in L^{\infty}(I, d \psi),
\end{gathered}
$$

and, by interpolation (see e.g. [23]),

$$
\|\hat{f} * \hat{g}\|_{p} \leq\|\hat{f}\|_{1} \cdot\|\hat{g}\|_{p} \quad \text { for every } \hat{f} \in L^{1}(I, d \psi), \hat{g} \in L^{p}(I, d \psi)(1<p<\infty) .
$$

In the following, * will always denote the convolution in the sense of (2.1). Set

$$
D_{N}=\sum_{0}^{N} \hat{\chi}_{n} \quad \text { and } \quad Q_{n}=\hat{\chi}_{n} /\left\|\hat{\chi}_{n}\right\|_{2}
$$

Since $P_{n}=\hat{\chi}_{n}[4]$, the $N$ th partial sum of the series expansion of $\hat{f} \in L^{p}(I, d \psi)$ with respect to the system $Q_{n}$ is

$$
S_{n} \hat{f}=D_{N} * \hat{f}=\sum_{0}^{N}\left(\int_{I} f Q_{n} d \psi\right) \cdot Q_{n} .
$$

Finally, $\left\|S_{N}\right\|_{p, p}$ will denote the norm of the linear operator $S_{N}$ from $L^{p}(I, d \psi)$ into itself.

3. For any $\hat{f} \in L^{p}(I, d \psi), F$ will denote the even function on $J=[-\pi, \pi]$ defined by

$$
F(\theta)= \begin{cases}\hat{f}(\theta) r \omega^{2} \pi^{-1}\left(r^{2}-\omega^{2} \cos ^{2}(\theta)\right)^{-1} & \text { if } \theta \in[0, \pi] \\ F(-\theta) & \text { if } \theta \in[-\pi, 0)\end{cases}
$$

We also set $d s=\sin ^{2}(\theta) d \theta$.

LEMMA. Let $\hat{f}$ and $F$ be defined as above, then

$$
F \in L^{p}(J, d s) \quad \text { and } \quad\|\hat{f}\|_{p} \cdot k_{p} \leq\|F\|_{L^{p}(J, d s)} \leq h_{p}\|\hat{f}\|_{p}
$$


where $k_{p}$ and $h_{p}$ are positive constants depending only on $p$ and on $r$. Moreover, for every $N>0$ we have

$$
\begin{aligned}
S_{N} \hat{f}= & \frac{a^{2}}{2} \sum_{0}^{N}\left(\int_{J} F Y_{n} d s\right) Y_{n}+\frac{b^{2}}{2} \sum_{0}^{N}\left(\int_{J} F Y_{n-2} d s\right) Y_{n-2} \\
& -a b \sum_{0}^{N-2}\left(\int_{J} F(\theta) \cos (2 \theta) Y_{n}(\theta) \sin ^{2}(\theta) d \theta\right) Y_{n} \\
& +\frac{a b}{2}\left(\left(\int_{J} F Y_{N-3} d s\right) Y_{N-1}+\left(\int_{J} F Y_{N-2} d s\right) Y_{N}\right) .
\end{aligned}
$$

PROOF. (3.2) is obvious from the definition of $F$ and the expression of $d \psi$. To prove (3.3) we observe that $\int_{0}^{\bar{\pi}} \hat{f} Q_{n} d \psi=(a / 2)\left(\int_{J} F Y_{n} d s\right)-(b / 2)\left(\int_{J} F Y_{n-2} d s\right)$ for every $n \geq 1$. Therefore (3.3) follows from straightforward calculation.

THEOREM. (i) If $3 / 2<p<3$ and $\hat{f} \in L^{p}(I, d \psi)$ then $\left\|S_{N} \hat{f}-\hat{f}\right\|_{p} \rightarrow 0$ as $N \rightarrow \infty$.

(ii) If $p \geq 3$ or $1 \leq p \leq 3 / 2$ then there exists a $G_{\delta}$ dense subset $H$ of $L^{p}(I, d \psi)$ such that $\lim \sup _{N}\left\|S_{N} \hat{f}\right\|_{p}=+\infty$ for every $\hat{f} \in H$.

(iii) If $p>3 / 2$ and $\hat{f} \in L^{p}(I, d \psi)$ then $S_{N} \hat{f}$ converges to $\hat{f}$ pointwise a.e. $[d \psi]$.

(iv) If $p \leq 3 / 2$ then there exists a $G_{\delta}$ dense subset $H^{\prime}$ of $L^{p}(I, d \psi)$ such that $S_{n} \hat{f}$ does not converge on any set of positive measure for every $\hat{f} \in H^{\prime}$.

Proof. Since the linear span of the $Q_{n}$ is dense in $L^{p}(I, d \psi)$, (i) and (ii) hold if and only if the norms $\left\|S_{N}\right\|_{p, p}$ are uniformly bounded, respectively, unbounded, as $N \rightarrow \infty$. By the definition of $S_{N}$, we also have

$$
\begin{aligned}
\int_{I} S_{N} \hat{f} \hat{\hat{g}} d \psi & =\int_{I} \sum_{0}^{N}\left(\int_{I} \hat{f} Q_{k} d \psi\right) Q_{k} \overline{\hat{g}} d \psi=\sum_{0}^{N}\left(\int_{I} \hat{f} Q_{k} d \psi\right)\left(\int_{I} Q_{k} \overline{\hat{g}} d \psi\right) \\
& =\int_{I} \hat{f} S_{N} \overline{\hat{g}} d \psi
\end{aligned}
$$

Hence,

$$
\left\|S_{N}\right\|_{p, p}=\left\|S_{N}\right\|_{q, q} \text { if } 1 / p+1 / q=1 .
$$

It is obvious from the expression of $d \psi$ that, if $\hat{g} \in L^{p}(I, d \psi)$,

$$
\|\hat{g}\|_{p} \cdot k_{p} \leq\left(\int_{J}|\hat{g}|^{p} d s\right)^{1 / p} \leq h_{p}\|\hat{g}\|_{p}
$$

where $k_{p}$ and $h_{p}$ are positive constants depending only on $p$ and $r$. Now, for $3 / 2<p<3$,

$$
\left\|\sum_{0}^{N}\left(\int_{J} \hat{g} Y_{n} d s\right) Y_{n}\right\|_{L^{p}(J, d s)}<\text { const }\|\hat{g}\|_{L^{p}(J, d s)} \quad \text { by Pollard [17]. }
$$

Moreover, one easily gets $\operatorname{Sup}_{n}\left\|Y_{n}\right\|_{L^{p}(J, d s)}<\infty$ for every $p<3$. Hence, (i) follows from (3.3) and (3.5). 
To prove (ii) observe that

$$
\begin{aligned}
\left\|Q_{n}\right\|_{p} \geq \mathrm{const} & \mid a\left(\int_{0}^{\pi / 2}|\sin (n+1) \theta|^{p} \theta^{2-p} d \theta\right)^{1 / p} \\
& \quad-b\left(\int_{0}^{\pi / 2}|\sin (n-1) \theta|^{p} \theta^{2-p} d \theta\right)^{1 / p}|=| A-B \mid
\end{aligned}
$$

and

$$
\begin{aligned}
& A^{p}=a^{p}(n+1)^{p-3} \sum_{0}^{n} \int_{k \pi / 2}^{(k+1) \pi / 2}|\sin \theta|^{p} \theta^{2-p} d \theta, \\
& B^{p}=b^{p}(n-1)^{p-3} \sum_{0}^{n-2} \int_{k \pi / 2}^{(k+1) \pi / 2}|\sin \theta|^{p} \theta^{2-p} d \theta .
\end{aligned}
$$

If we set $\alpha_{p, n}=\sum_{0}^{n} \int_{k \pi / 2}^{(k+1) \pi / 2}|\sin \theta|^{p} \theta^{2-p} d \theta$ we get, since $a>b$,

$$
\begin{aligned}
& \left\|Q_{n}\right\|_{3} \geq \operatorname{const}\left(a \alpha_{3, n}^{1 / 3}-b \alpha_{3, n-2}^{1 / 3}\right) \geq \operatorname{const}(\log n)^{1 / 3} \quad \text { if } p=3 \\
& \left\|Q_{n}\right\|_{p} \geq \text { const } a\left\{(n+1)^{1-3 / p} \alpha_{p, n}^{1 / p}-b(n-1)^{1-3 / p} \alpha_{p, n-2}^{1 / p}\right\} \geq \text { const } n^{1-3 / p}
\end{aligned}
$$

Therefore $\left\|Q_{n}\right\|_{p} \rightarrow+\infty(n \rightarrow \infty)$ for $p>3$.

Choose now $\hat{f}_{n}=2\left(\cos ((n+2) \theta)-\omega^{-2} \cos (n \theta)\right)=a^{-1}\left(Q_{n+2}-Q_{n}\right)$. We have $\left\|\hat{f}_{n}\right\|_{p}<4 r \omega^{-2}$ and $\left\|S_{n} \hat{f}_{n}\right\|_{p}=a^{-1}\left\|Q_{n}\right\|_{p}$. Hence, (ii) follows from (3.6) and (3.4).

Assertion (iii) follows from (3.3). Namely, if $f \in L^{p}(I, d \psi)$, then $F(\theta)$ and $F(\theta) \cos \theta \in L^{p}(J, d s)$ and, if $p>3 / 2, \int_{J} F Y_{n} d s \rightarrow 0$ for $n \rightarrow \infty$. In fact, $F(\theta) \sin \theta \in L^{p^{\prime}}(T)$ for some $p^{\prime}>1$ with $p^{\prime}(3-p)<p$ and

$$
\int_{J} F(\theta) Y_{n}(\theta) \sin ^{2} \theta d \theta=\int_{-\pi}^{\pi}\{F(\theta) \sin \theta\} \sin ((n+1) \theta) d \theta \rightarrow 0 \text { for } n \rightarrow \infty .
$$

Then, by the Carleson-Hunt Theorem [12], the right-hand side of (3.3) tends to $\left(\left(a^{2}+b^{2}\right) / 2-a b \cdot \cos (2 \theta)\right) \pi F(\theta)=\hat{f}(\theta)$.

If $p \leq 3 / 2$ then, by (3.6) and the uniform boundedness theorem, there exists a $G_{\delta}$ dense subset $H^{\prime} \subset L^{p}(I, d \psi)$ such that for every $\hat{f} \in H^{\prime}$ we have

$$
\operatorname{Sup}_{n}\left|\int_{I} Q_{n} \hat{f} d \psi\right|=+\infty
$$

If $\hat{f} \in H^{\prime}$ then $S_{n} \hat{f}$ does not converge pointwise a.e. on any set of positive measure. In fact, suppose, by way of contradiction, that $S_{n} \hat{f}$ converges a.e. on a set $E$ of positive measure. Then

$$
\left|S_{n} \hat{f}(x)-S_{n-1} \hat{f}(x)\right|=\left|\left(\int_{I} \hat{f} Q_{n} d \psi\right) Q_{n}(x)\right| \rightarrow 0 \quad \text { a.e. on } E .
$$


Arguing as in the proof of the Cantor-Lebesgue Theorem [23] we shall show that (3.8) implies $\int_{I} \hat{f} Q_{n} d \psi \rightarrow 0$, which, by (3.7), is a contradiction. In fact, one has

$$
\begin{aligned}
\int_{E}\left|Q_{n}\right|^{2} d \theta & \geq \int_{E}|a \cdot \sin ((n+1) \theta)-b \cdot \sin ((n-1) \theta)|^{2} d \theta \\
& =\left\{\left(a^{2}+b^{2}\right) 2^{-1}\right\} m(E)-a b \int_{E} \cos (2 \theta) d \theta+o(1)
\end{aligned}
$$

and, since $\left(a^{2}+b^{2}\right) 2^{-1}-a b>0, Q_{n}$ cannot tend to zero a.e. on $E$. This concludes the proof of the Theorem.

REMARKS. (1) The spaces $L^{p}(I, d \psi)(1<p<\infty)$ can be interpreted as spaces of radial functions on $F_{r}$. In fact, if $\Gamma=\left(L^{2}\left(F_{r}\right), V N\left(F_{r}\right), m\right)$ denotes the standard dual gage of $F_{r}$, then $L^{p}(I, d \psi)(1<p<\infty)$ is isometrically isomorphic to the subspace $L_{R}^{p}(\Gamma)$ of $L^{p}(\Gamma)$ generated by the finitely supported radial functions (see e.g. $[8,13,22])$.

A function $f$ defined on $F_{r}$ is a multiplier of $L^{p}(\Gamma)$ into itself if there exists a bounded linear operator $M_{f}: L^{p}(\Gamma) \rightarrow L^{p}(\Gamma)$ such that $M_{f} T_{\psi}=T_{f \psi}$ whenever $\psi$ is a finitely supported function and $T_{g}$ denotes the left convolution operator by $g$ on $l^{2}\left(F_{r}\right)$. By (i) and (ii), $L_{R}^{p}(\Gamma)$ has an approximate unit which is bounded in the multiplier norm if $3 / 2<p<3$, while if $p \leq 3 / 2$ or $p \geq 3$ the multiplier norm of the functions $f_{N}=\sum_{0}^{N} \chi_{k}$ is unbounded (compare [11]). The same technique used to prove (ii) shows also that no radial function $f=\sum_{1}^{\infty} a_{n} \chi_{n}$ such that $a_{n_{1}}=a_{n_{2}}=\cdots=0$ for some subsequence $\left\{n_{j}\right\}$ and such that $a_{n}(\log n)^{1 / 3} \rightarrow \infty$, respectively, $a_{n}(n)^{1-3 / p} \rightarrow \infty$, can be a multiplier of $L^{3}(\Gamma)$, respectively, of $L^{p}(\Gamma)$ with $p>3$.

(2) Betori and Pagliacci [2] have recently extended the results of Figà-Talamanca and Picardello [9] to the context of groups acting faithfully and simply transitively on an homogeneous tree of order $s$. We notice that our Theorem holds also for such groups.

\section{REFERENCES}

1. J. P. Arnaud, Fonctions sphériques définies sur arbre homogène, C. R. Acad. Sci. Paris 290 (1980), 99-101.

2. W. Betori and M. Pagliacci, Harmonic analysis for groups on trees, preprint

3. P. Cartier, Harmonic analysis on trees, Proc. Sympos. Pure Math., vol. 26, Amer. Math. Soc., Providence, R.I., 1972, pp. 419-424.

4. J. M. Cohen, Operator norms on free groups, Boll. Un. Mat. Ital. B (6) 1 (1982), 1055-1065.

5. J. M. Cohen and L. De Michele, The radial Fourier-Stieltjes algebra of free groups, Contemporary Math., Vol. 10, Amer. Math. Soc., Providence, R.I., 1982, pp. 33-40.

6. B. Dreseler and P. M. Soardi, A Cohen type inequality for uttraspherical series, Arch. Math. (Basel) 38 (1982), 243-247.

7. A. Erdelyi, Higher tracendental functions, Vols. I-III, Bateman Manuscript Project, McGrawHill, New York, 1955.

8. P. Eymard, L'algèbre de Fourier d'un groupe localement compact, Bull. Soc. Math. France 92 (1964), 181-236.

9. A. Figà-Talamanca and M. Picardello, Spherical functions and harmonic analysis on free groups, J. Funct. Anal. 47 (1982), 281-304.

10. __ Harmonic analysis on free groups, Lecture Notes in Pure and Appl. Math., vol. 87, Dekker, New York, 1983.

11. U. Haagerup, An example of a nonnuclear $C^{*}$ algebra which has the metric approximation property, Invent. Math. 50 (1979), 279-293. 
12. R. Hunt, On the convergence of Fourier series, Proc. Conf. Orthogonal Expansions and Their Continuous Analogues, Carbondale Press.

13. R. A. Kunze, $L_{p}$ Fourier transforms on locally compact unimodular groups, Trans. Amer.Math. Soc. 89 (1958), 519-540.

14. A. Iozzi and M. Picardello, Spherical functions on graphs, preprint.

15. F. Mayer-Lindenberg, Zur Dualitätstheorie symmetrischer Paare, Crelle's J. 321 (1981), 36-52.

16. C. Meaney, Almost everywhere divergent Jacobi polynomial series, preprint.

17. H. Pollard, The mean convergence of orthogonal series. II, Trans. Amer. Math. Soc. 63 (1948), 355-367.

18. __ The mean convergence of orthogonal series. III, Duke Math. J. 16 (1949), 189-191.

19. __ The convergence almost everywhere of Legendre series, Proc. Amer. Math. Soc. 35 (1972), 442-444.

20. T. Pytlik, Radial functions on free groups and a decomposition of the regular representation into irreducible components, J. Reine Angew. Math. 326 (1981), 124-135.

21. R. Stanton and P. A. Thomas, Polyhedral summability of Fourier series on compact Lie groups, Amer. J. Math. 100 (1978), 477-493.

22. W. Stinespring, Integration theorems for gages and duality for unimodular groups, Trans. Amer. Math. Soc. 90 (1959), 15-56.

23. A. Zygmund, Trigonometric series. Vols I, II, 2nd ed., Cambridge Univ. Press, London and New York, 1959.

Istituto Matematico, Universita di Milano, Via C. Saldini 50, 20133 Milano, ITALY 